\title{
Toward Micro Wall-Climbing Robots Using Biomimetic Fibrillar Adhesives
}

\author{
Matthias Greuter ${ }^{1}$, Gaurav Shah ${ }^{2}$, Gilles Caprari ${ }^{1}$, Fabien Tâche ${ }^{1}$, Roland \\ Siegwart ${ }^{1}$, Metin Sitti ${ }^{2,3}$ \\ ${ }^{1}$ Autonomous Systems Lab (ASL), École Polytechnique Fédérale de \\ Lausanne (EPFL), \{name.familyname\}@epfl.ch \\ ${ }^{2}$ Dept. of Mechanical Engineering, Carnegie Mellon University (CMU), \\ gshah@cmu.edu \\ ${ }^{3}$ Robotics Institute, Carnegie Mellon University, sitti@cmu.edu
}

\begin{abstract}
Climbing is a challenging task for autonomous mobile robots primarily due to requirements for agile locomotion, and high maneuverability as well as robust and efficient attachment and detachment. A novel miniature wall-climbing robot is proposed. The robot is adapted for the wall-climbing task by taking advantage of down scaling and its low design. Challenges encountered during robot miniaturization and performances of the robot are reported. The miniature robot prototype proved to be able to climb on inclined surfaces with a slope of up to $90^{\circ}$ at a speed of $3.3 \mathrm{~mm} / \mathrm{s}$. It is equipped with sensors that enable it to avoid obstacles, follow walls and detect free-falls. It can be controlled by remote control or act autonomously. Animals, such as Geckos, have developed amazing climbing ability through micro- and nano-fibers on their feet. These structures have inspired the study of dry adhesion and the design of synthetic fibrillar pads presented in the paper.
\end{abstract}

\section{Introduction}

Research and development of wall-climbing robots has been pursued for years. The ability of these robots to go over obstacles greater than their size makes them suitable for a wide variety of applications. Wall-climbing robots are very useful for repair and maintenance in environments inaccessible or hazardous to humans. Some applications for which wall-climbing robots have been researched and developed are: inspecting nuclear power plants [1]; cleaning [2]; sand blasting [3]; search and rescue, and toys. 
Study of existing wall-climbing robots indicates that the major challenges still impeding their development are high adhesion, efficient detachment, and rough surface adaptation. Dry adhesion, such as biomimetic fibrillar adhesives, enjoys a number of advantages over other natural and commercial adhesion mechanisms. The paper presents a detailed analysis of a novel miniature wall-climbing robot, using dry adhesives.

\section{Wall-climbing Robot Design}

The research aims at the design of a miniature wall-climbing robot. Miniaturization of the robot is limited by current technology and affordability. The aim is to design a robot in the same order of magnitude as the Alice robot developed at EPFL [4]. The robot should occupy a volume around $104 \mathrm{~mm} 3$ and weight less than $11 \mathrm{~g}$. These specifications are close to the theoretical minimum possible with components available today. The presented robot design is based on the Alice robot and hence inherits all its functions.

In order to achieve efficient wall-climbing the robot should be able to change orientation and climb in all directions. The ability to climb at reasonable speeds (at least $5 \mathrm{~mm} / \mathrm{s}$ ) and avoid obstacles along with knowledge about current configuration is desired. For autonomous performance, the source of energy, microprocessor, actuators and sensors have to be placed on the robot. The ability to adapt the robot to different tasks can result from its modularity and the possibility to reprogram its software. To achieve a high work time ( $>5 \mathrm{~h})$, the power consumption has to be minimized $(<20 \mathrm{~mW})$. The robot design is described and analyzed in detail in the following sections.

\subsection{Force Calculations}
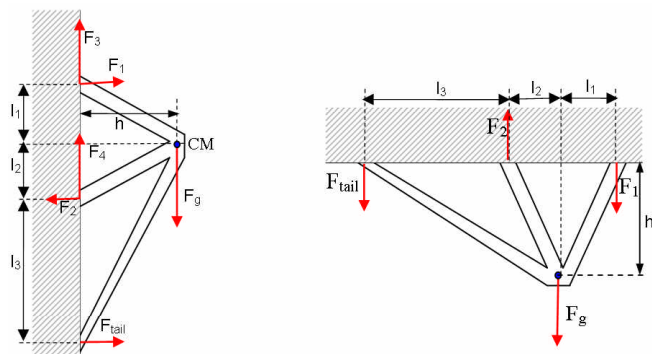

Fig. 1. a) Forces on robot going upward. b) walking upside down. 
The wall-climbing robot model presented in [5] is used as the basis for the robot design proposed in this paper. The application of static equilibrium equations to the wall-climbing robot model in various wall-climbing modes shown in Fig. 1 gives Eq. (1)-(4). These are used to find a favorable form for the robot and determine suitable dimensions.

For the robot walking upward (Fig. 1 a):

$$
F_{1}=\left(-F_{g} h-F_{2} l_{2}+\left(l_{2}+l_{3}\right) F_{\text {tail }}\right) / l_{1} \quad / / \quad F_{2}=\left(-F_{g} h-F_{1} l_{1}+\left(l_{2}+l_{3}\right) F_{\text {tail }}\right) / l_{2}
$$

For the robot walking downward:

$$
F_{1}=\left(F_{g} h-F_{2} l_{2}+\left(l_{2}+l_{3}\right) F_{\text {tail }}\right) / l_{1} \quad / / \quad F_{2}=\left(F_{g} h-F_{1} l_{1}+\left(l_{2}+l_{3}\right) F_{\text {tail }}\right) / l_{2}
$$

Note that the difference of the Eqs. (1) is $2 F_{g} h / l_{l}$, and the difference in (2) is $2 F_{g} h / l_{2}$.

For the robot walking sideways:

$$
2 \cdot F_{a} \cdot l_{a}=F_{g} \cdot h \quad / / \quad F_{a}=F_{g} \cdot h / 2 l_{a}=m \cdot g \cdot h / 2 l_{a}<F_{\text {adhesion }}
$$

For the robot walking upside down (Fig. $1 \mathrm{~b}$ ):

$$
\begin{gathered}
F_{1} l_{1}+F_{2} l_{2}-F_{\text {tail }}\left(l_{2}+l_{3}\right)+F_{g} \cdot 0=0 \\
\left(F_{\text {preload }}\left(l_{1}+l_{2}\right)+F_{g} l_{2}\right) /\left(2 l_{2}+l_{3}\right)<F_{\text {tail }}<\left(F_{\text {adhesion }}\left(l_{1}+l_{2}\right)+F_{g} l_{1}\right) /\left(l_{1}+l_{2}+l_{3}\right)
\end{gathered}
$$

Since the robot will move in all directions, including up and down, the forces $F_{1}$ and $F_{2}$ should be as independent of gravity as possible. This implies that the mass $\mathrm{m}$ and the height of the center of mass have to be small. Thus the robot has to be designed to be light and low. The force $F_{l}$, provides the preload pressure necessary for good adhesion. Ideally the force $F_{2}$ should be equal and opposite of the adhesion force to facilitate easy detachment without additional force.

While the robot climbs upwards, $F_{l}$ is the limiting factor since gravity tends to minimize this force. If the robot climbs downwards, it risks detachment due to the additional force on $F_{2}$ by gravity. The robot is able to climb if the force the tail delivers is in the following range:

$$
\left(F_{\text {preload }}\left(l_{1}+l_{2}\right)+F_{g} h\right) / l_{3}<F_{\text {tail }}<\left(F_{\text {adhesion }}\left(l_{1}+l_{2}\right)-F_{g} h\right) /\left(l_{1}+l_{2}+l_{3}\right)
$$

This is only possible if the following condition is met:

$$
F_{\text {preload }}<\left\lfloor F_{\text {adhesion }} l_{3}-F_{g} h\left(l_{1}+2 l_{2}+l_{3}\right) /\left(l_{1}+l_{2}\right)\right\rfloor /\left(l_{1}+l_{2}+l_{3}\right)
$$

It follows that $F_{2},\left(l_{l}+l_{2}\right)$, and $l_{3}$ have to be maximized, and $F_{1}, F_{g}$ and $h$ have to be minimized. In other words, the robot should be long and equipped with a long tail, while maintaining low weight and height.

\subsection{Miniature Robot Design}

Legs, wheels, and tread based locomotion systems along with combinations of these have been used in climbing robots. The main advantage in utilizing tread-based locomotion mechanisms is that a big surface of adhesive can be in contact with the wall but only a small part of it has to be pre- 
loaded and peeled of at each instant. As mentioned before, it is essential that the robot is light. This criterion dominates the selection of components for the robot. The battery used for the robot is the Lithium-Polymer battery (Kok40, Kokam Inc.) which is very thin $(30 \mathrm{~mm}$ x $43 \mathrm{~mm}$ x $0.8 \mathrm{~mm}$ ). This form is ideal for a climbing robot. Its mass is only $1.7 \mathrm{~g}$ for a capacity of $40 \mathrm{mAh}$. To increase the reliability of localization by odometry, a dualaxis accelerometer is added. It provides information about the orientation of the robot. In addition, it can detect when the robot falls of the wall. Distance sensors allow the robot to avoid obstacles and follow walls. The robot can be controlled by a remote control or take decisions autonomously.

The actuators have to be as small and light as possible, have low energy consumption, have a high enough torque, turn in the right range of speed, and be easy to implement. Even though DC motors with a diameter as small as $4 \mathrm{~mm}$ and a length of $8 \mathrm{~mm}$ exist, these motors do have certain drawbacks such as high turning speed and low torque that make them unusable. Use of gears is hardly feasible due to the space limitations. Furthermore, encoders should be added for speed and position control. Watch step motors, as used on Alice seem to be advantageous.

\section{Prototype}
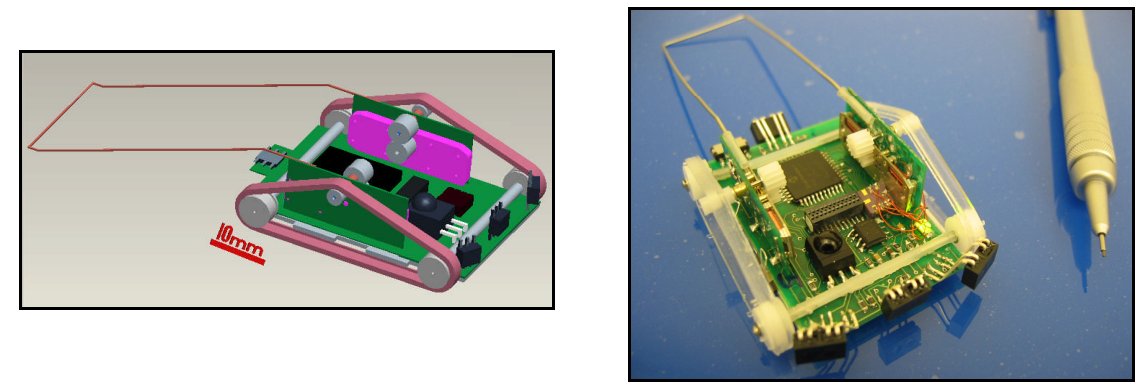

Fig. 2. CAD drawing (a) and snap (b) of the prototype.

To test the design, a prototype has been built (Fig. 2). The PCB designed for the climbing robot allows adding a PIC microcontroller, three distance sensors in the front to detect obstacles, an IR receiver for remote control, an accelerometer to calculate the robot's orientation and detect if it falls, and a connector to add modules. A $40 \mathrm{mAh}$ lithium-polymer battery is used to power the robot. The robot dimensions are about $40 \mathrm{~mm} \times 43$ $\mathrm{mm} \times 14 \mathrm{~mm}$ (without the tail), its mass is about $10 \mathrm{~g}$, and the centre of mass $(\mathrm{CM})$ is about $5 \mathrm{~mm}$ above ground. The adhesion force is provided by a belt made out of silicone rubber (Sylgard 184, mixing ratio10.7:1). 


\subsection{Adhesion force of the belt}

The current robot design uses silicone rubber belts for adhering to the surface. The surface of the molded belts is rough since the mold surface is machined on the mill. Fig. 3 shows that the adhesion force depends strongly on the surface quality. The values above the straight line represent the space in which the adhesion pressure is greater than the preload pressure, which is essential for the functioning of the robot.

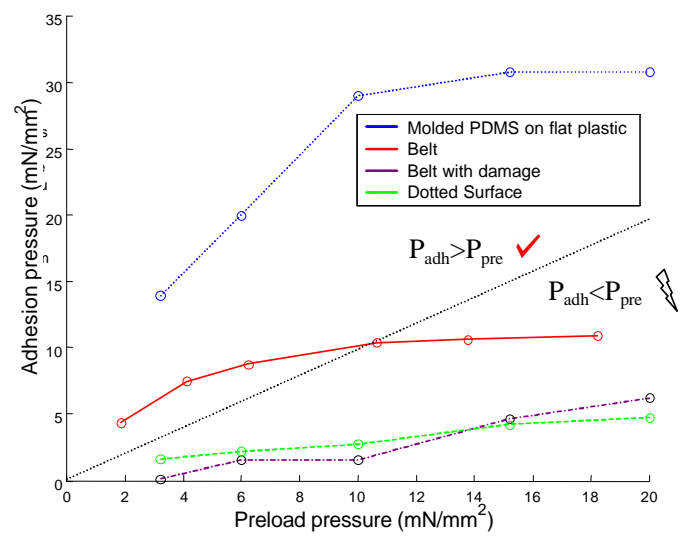

Fig. 3. Adhesion pressure of materials with different surface qualities in function of the preload pressure

\subsection{Torque Requirement}

The watch motor used on Alice [4] has a gear with a reduction of 180 . Its torque is 10 times bigger than the one of a pager DC motor and it turns about once per second which is a suitable speed for the robot. However, it needs to be verified if the motor provides sufficient torque to support the wall-climbing task. The calculations of the necessary motor torque showed that this is about $0.56 \mathrm{mNm}$. The torque of the ETA/Swatch motors used in Alice at $3 \mathrm{~V}$ is about $0.35 \mathrm{mNm}$ each. In the new design, another ETA/Swatch component is used, on which two independent rotors act on the same axis of the motor. Using these motors at $3.5 \mathrm{~V}$, the torque will be about twice the torque of Alice. Furthermore a reduction of $3 / 4$ is added. This gives a total useful torque of $1.8 \mathrm{mNm}$ for the robot. The motors deliver about three times the torque theoretically required by the robot. 


\subsection{Results}

The prototype was tested on Plexiglas. Good climbing ability was observed. The robot is able to climb upward on a surface inclined up to $90^{\circ}$, walk downwards on a surface orientated at $85^{\circ}$ and walk sideways on a surface inclined at $87^{\circ}$. Its speed is about $3.3 \mathrm{~mm} / \mathrm{s}$.

The main problem identified is that the belt made out of silicone rubber does not have a high enough adhesion force and the robot is slipping and gliding on the surface. The belt is not self cleaning and the adhesion force diminishes more with time since it gets dirty.

Using Eq. (1)-(4) and the dimensions of the prototype, it can be calculated that the preload pressure of the belt should be around $6 \mathrm{KPa}$ and adhesion pressure required is around $20 \mathrm{KPa}$. Observing Fig. 3, it can be recognized that this is not easily achievable using rubber belts. In the next section we explore the feasibility of replacing the rubber belt with synthetic fibrillar adhesive tape inspired by gecko-feet structure.

\section{Biomimetic Dry Adhesion}

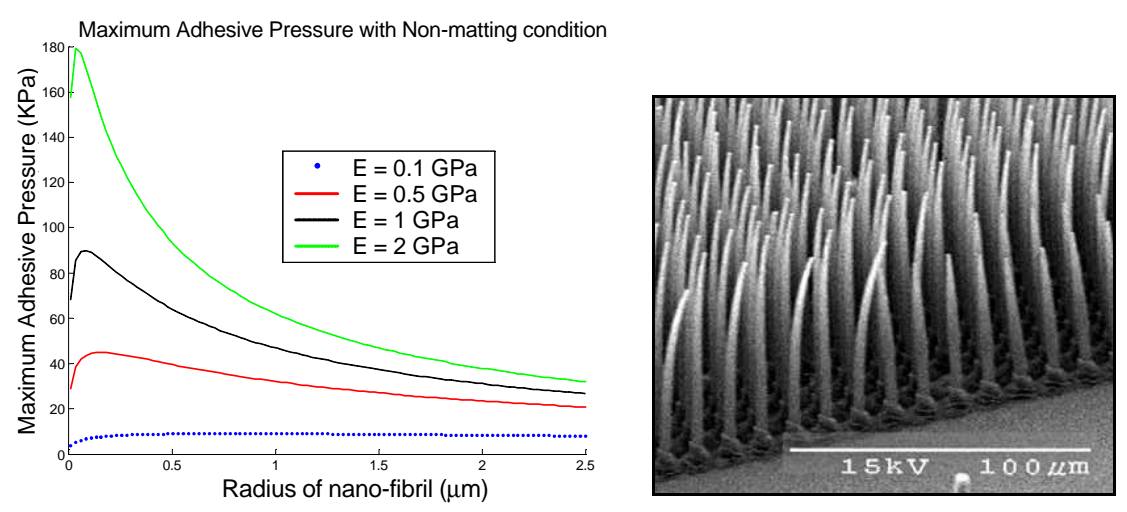

Fig. 4. Influence of fiber geometry and material on maximum adhesive pressure;

Synthetic 2 micron diameter fibers

Dry adhesion, using synthetic fibrillar adhesives (Fig. 4), is a promising adhesion mechanism for the robot design proposed above. Like the geckofeet, the synthetic adhesives are covered with oriented nano-fibers with aspect ratios as high as 1:30. These nano-fibers provide millions of compliant points of contact for adhesion to rough surfaces. Dry adhesion is based on the van der Waals intermolecular forces present at the fiber-surface points of contact [7]. These forces exist between the substrate and each individual fiber, together resulting in a very high adhesion force [6]. 
The fibers need to be packed as closely as possible in synthetic adhesives for high adhesion [9]. However, the density of the fibers is limited by their tendency to stick to each other and get entangled, known as matting. The matting condition depends on the fiber material properties and geometry. The matting condition determines the density for different fibers and hence the adhesion. Fig. 4 plots the maximum adhesive pressure and indicates the strong dependence of adhesion on material properties and fiber geometry. It is evident from Fig. 4 that for high adhesion the fibers need to be fabricated from a stiff polymer with small radii $(<200 \mathrm{~nm})$.

\subsection{Rough Surface Adaptation}

One of the major advantages of fibrillar adhesives is their ability to adapt to rough surfaces. For solid adhesives the presence of dirt on the substrate and the substrate roughness lead to contacts that are not robust. As stated before, it is quite evident that the total adhesive force of an adhesive pad depends on the number of hairs in contact. In order to achieve good contact between the fibril and the substrate it is necessary that the fiber array is compliant. One way to achieve this is by using soft polymers for fiber fabrication. However, this will significantly reduce the fiber density and hence the adhesive pressure, due to the matting condition. The smart way to achieve enhanced fiber compliance and also maintain high fiber density is to use oriented fibers. Orienting the fibers significantly enhances their compliance in the normal (to surface) direction and hence also their rough surface adaptability. This is due to the fact that high aspect ratio fibers are very compliant in the lateral direction as compared to the axial direction [8]. Surface roughness has a significant effect on the preload required for good contact and the required adhesion. The applied preload for attaching an adhesive sample to a rough surface results in fiber deformation in the form of compression, bending, and buckling. For thin slender fibers, modeled as cantilever beams, the fiber deformation from bending and buckling is much greater than the axial compression. The miniature robot is designed for the use of the fibrillar adhesives. The belts, now molded of silicon rubber, can easily be exchanged by belts using the fibrillar adhesive.

\section{Conclusion}

Miniature wall-climbing robots have a number of advantages over their larger counterparts. The miniature wall-climbing robot design presented in the paper overcomes challenges in miniaturization and demonstrates effec- 
tive climbing behavior on inclined surfaces with a slope of up to $90^{\circ}$ at a speed of $3.3 \mathrm{~mm} / \mathrm{s}$. The robot design is demonstrated to be efficient, reliable, and robust. The robot is capable of intelligent and autonomous navigation.

Future work includes improving the quality of the belts in using biomimetic fibrillar adhesives, increasing motor torque, refining the transmission, and optimizing the use of the accelerometer. New modules can be added to satisfy different scenarios. The presented research opens a new avenue in the design of high performance miniature wall-climbing robots using biomimetic fibrillar adhesives.

\section{References}

1. L. Briones, P. Bustamante, and M. Serna (1994) ROBICEN: A wall-climbing pneumatic robot for inspection in nuclear power plants. Robotics and Computer-Integrated Manufacturing 11, 287-292

2. Z. Yanzeng, S. Hao, and W. Yan (1999) Wall-climbing robot with negative pressure sucker used for cleaning work. High Technology Letters 5, 85-88

3. L. Shuliang, Z. Yanzheng, G. Xueshan, X. Dianguo, Wang Yan (2000) A wall-climbing robot with magnetic crawlers for sand-blasting. Spray-painting and measurement High Technology Letters 10, 86-8

4. G. Caprari, T. Estier, R. Siegwart (2002) Fascination of Down Scaling - Alice the Sugar Cube Robot. J. of Micro-Mechatronics, VSP, Vol. 1, No. 3, 177-189

5. C. Menon, M. Murphy, M. Sitti (2004) Gecko Inspired Surface Climbing Robots. IEEE Int. Conf. on Robotics and Biomimetics (ROBIO), China, pp. 3-5

6. G. Shah, M. Sitti (2004) Modeling and Design of Biomimetic Adhesives Inspired by Gecko Foot-Hairs. IEEE Int. Conf. on Robotics and Biomimetics (ROBIO), China

7. K. Autumn, et al (2002) Evidence for Van der Waals Attachment for Geckos. Proc. Nat. Acad. Science, vol. 99, no. 19, pp. 12252-12256

8. M. Sitti, R. S. Fearing (2003) Synthetic gecko foot-hair micro/nano-structures as dry adhesives. J. of Adhesion Science and Technology, vol. 17, no. 8, pp. 1055-1074

9. C.-Y. Hui, N. J. Glassmaker, T. Tang, A. Jagota (2004) Design of biomimetic fibrillar interfaces: 2. Mechanics of enhanced adhesion. J. R. Soc. Lond. Interface, $1-14$ 